\title{
La bibliotecología y la sociedad de la información: algunos aspectos a considerar en torno a las prácticas lectoras
}

\author{
ElSA MARgaRita RamíRez Leyva \\ Centro Universitario de Investigaciones Bibliotecológicas \\ Universidad Nacional Autónoma de México \\ 04510, México, D.F., Tel. 56-23-03-29 \\ E-mail: eramirez@ servidor.unam.mx
}

Trabajo recibido

el 26 de abril de

1999

Trabajo aceptado el 28 de mayo de 1999

\section{RESUMEN}

Algunas de las características de la llamada sociedad de la información en donde la tecnología constituye su base material, la información su recurso, la educación el medio para lograr desarrollo y bienestar social, se relacionan con el ámbito bibliotecológico, concretamente en lo referente a prácticas de lectura y de información, en las que se plantean transformaciones, así como el surgimiento de nuevas modalidades de comportamiento a partir de documentos y servicios digitales que empiezan a crearse con cánones diferentes a los establecidos por el medio impreso. A partir de ellos surgen también significadosy símbolos que han empezados a introducirse en el entramando social de manera desigual dadas las diferentes capacidades de lectura y de recursos para acceder a los nuevos medios, lo que determina a las posibilidades para renovar los conocimientos de los individuos o bien los limitan y reducen el aprovechamiento de la creciente oferta informativa para diversas actividades que dependen cadavez más deesa ofertay de la capacidad delos lectores para su aprovechamiento.

\section{LIBRARY SCIENCE AND THE INFORMATION SOCIETY: ASPECTS TO CONSIDER WTTH REGARD TO READING PRACTICES ELSA MARGARITA RAMÍREZ-LEYVA}

\section{ABSTRACT}

Some of the characteristics of the so called "information society", in which technology constitutes its material base, information its resource, and education the means through which social change and well being are achieved, are related to the field of Library Science, especially with regard to reading practices and information in which transformations are posited, as well as with the emergence of new behavioral modalities that are the result of documents and digital services that begin to impose rules others than those established by print media. These signifiers and symbols, as a result the varying degrees of access and ability of users, are emerging in the social fabric and serve both to open and, conversely, access ways close- when reading capacity is limited- to the growing supply of information available. 
...una caracteística exinial denuestra soiedades una coxistenia desada etreuma tendoǵa mynuearyunas formassoialesmyvigas

Raymond Williams

\section{LA NUEVA TECNOLOGÍA DE LA INFORMACIÓN Y SU SIGNIFICACIÓN SO- CIAL EN EL ÁMBITO BIBLIOTECOLÓGICO}

T os fenómenos relacionados con el comportamiento humano son complejos de ـbido a la diversidad de variables que participan en él, tanto las que son propias del ser individual, como las del ser social. Por ello, es necesario estudiar dichos fenómenos desde diferentes perspectivas (sociológica, psicológica, antropológica, bibliotecológica, jurídica e histórica entre otras).

La bibliotecología, de acuerdo con el paradigma aún vigente-proteger ante todo el interés público y el libre acceso a la información, considerada ésta más como un servicio que como una mercancía- , se centra en el estudio de las formas, medios, flujos, relaciones, conductas y significados involucrados en el registro, organización, comunicación y uso de los conocimientos que producen los diferentes sectores dela sociedad, con la finalidad de comprender las condiciones humanas, materiales y tecnológicas, que determinan las peculiaridades de las prácticas de lectura y de información y que nos permiten caracterizar las necesidades de los diferentes públicos, que son la base para formular elementos generalizadores de los principios de relevancia, oportunidad, accesibilidad y disponibilidad de la información registrada. Ahora, con el apoyo tecnológico, a los principios antes señalados deberán incorporarse las cualidades de flexibilidad, interconectividad e interactividad para adaptarlos a individualidades y a necesidades muy específicas.

Así, la tecnología de la información plantea un nuevo fenómeno, no sólo para la bibliotecología sino también para otras disciplinas, como medio de registro de información y como medio de comunicación con el que se establecen significaciones, relaciones y funciones de particular complejidad debido a la interacción que se entabla con una composición social de suyo complicada, dada la diversidad de la naturaleza humana. La interacción con la información, que renueva el conocimiento, puede, por lo tanto, producir diferentes efectos. Las características de esta nueva tecnología - quevehiculiza la información-, empiezan a tomar distancia de las que dieron lugar a la cultura impresa y son incorporadas al igual que las expresiones 
audiovisuales de las que están surgiendo los medios hipertextuales y la multimedia, como afirma Castells:

[...]la revolución tecnológica, centrada en tomo a las tecnologías de la información, está modificando la base material de la sociedad a un ritmo acelerado... Además un nuevo sistema de comunicación, que cada vez habla más un lenguaje digital universal, está integrando globalmente la producción y distribución de palabras, sonidos e imágenes de nuestra cultura, y acomodándolas a los gustos de las identidades y temperamentos de los individuos. Las redes informáticas interactivas crecen de modo exponencial, creando nuevas formas y canales de comunicación, y dando forma a la vida a la vez que ésta les da forma a ellas. ${ }^{1}$

Es decir, estamos ante una innovación tecnológica que, según se vislumbra, transformará las estructuras sociales y desde luego las mentalidades. Así pues, la tecnología de la información es parte de la denominada cultura cibernética y aunque esta tecnología todavía se encuentra en su fase embrionaria, ya se pueden identificar en torno a ella objetos y símbolos relacionados con la computadora y los medios digitales a los que cada vez se agregan más tareas. A simismo, ya se pueden advertir relaciones interpersonales a través de las redes electrónicas, lo cual constituye una nueva forma de socialización que, por ahora, sólo se da en un segmento culto y económicamente dotado para acceder a tal tecnología, pero tiende a extenderse a otros sectores de la sociedad.

Todo ello conduce a formular significadosy símbolos que se integran a las identidades individuales y sociales en cuya contextualización participan diversas instituciones. Aunado a la idea de Strathern sobre la contextualización de la tecnología en los ambientes domésticos, es decir en el hogar, cabría agregar la biblioteca y la universidad y por supuesto otros ambientes laborales. En estos espacios se contextualiza "el 'comportamiento' de los ingenios tecnológicos como objetos que habitan las casas, ocupan un lugar en el espacio doméstico y son parte dinámica de la vida cotidiana y de las relaciones familiares." 2

En este sentido, en el ámbito bibliotecario encontramos los primeros usos de la computadora para el proceso de registros documentales, así como los cambios en los servicios y productos de información y en los métodos para su desarrollo y servicio al público. D e esta manera, desde los años sesenta en los Estados Unidos y en algunos países de Europa y una década más tarde en México y en algunas bibliotecas latinoamericanas, la biblioteca contribuye de forma importante a la implantación de la nueva tecnología en el procesamiento de la información y al uso de la computadora para acceder a la información en línea.

1 Manuel Castells. Laeradelainfomadón: Economá, soiedadyaultura, vol. 1, Lasocedadreel pp. 27 y 28.

2 Roger y Hirsch Silverstone. Eds. Losfetosdela nueva commicacón: e consumodelanuevateendoǵaend hogaryla familia p. 13. 


\section{InnestigacónBiblictedógica V. 13 No. 26 enero/ junio de 1999}

Hoy, en los albores del siglo XX I, la inserción de la tecnología en el manejo y uso de la información en las bibliotecas de nuestra región es aún desigual, pese a que la información ha adquirido una significación cada vez más destacada en amplios ámbitos sociales de países con economías desarrolladas, que la consideran un recurso estratégico, al grado que D ormido, Morales y Abad, ${ }^{3}$ piensan que la energía y la ma teria han pasado a un segundo plano en la investigación científica, en tanto quelainformación y el conocimiento se han convertido en el nuevo objeto formal de la ciencia y la tecnología. Prueba de ello es que la economía, la cultura y el bienestar dependen cada vez más del nivel alcanzado en el campo de las llamadas altas tecnologías de información. Como afirman estos autores, "su presencia avivará el suministro inagotable del conocimiento mismo." 4

Esto nos lleva a pensar que el statusde la información ha cambiado gracias a los nuevos soportes en que se registra y comunica, es decir, gracias a la tecnología de la información; sin embargo, no podemos ignorar que la información se ha constituido en la mercancía de una industria cada vez más poderosa, a grado tal que está desarrollando formas de consumo que abarcan diversas actividades de los individuos, incluso con productos y servicios novedosos y atractivos para los sectores proclives al consumismo innovador, sea por que lo existente les resulta insuficiente o simplemente por integrarse a las clases que buscan distinguirse del resto de la población y mantener su nivel de elite. Incluso se podría pensar en un paralelismo con la aparición del libro y posteriormente del invento de G utenberg, que dio lugar a la revolución tipográfica. ${ }^{5} \mathrm{D}$ el mismo modo que durante laE dad Mediay el Renacimiento las sociedades se organizaban en torno de la escritura y luego de la palabra impresa en el formato códex - que dio paso al libro- , ahora la sociedad cada vez más se organiza en torno de los sistemas basados en la pantalla y en el texto digital, donde el conocimiento de otra época y de la actual ha sido legitimado por grupos de poder y por intelectuales; desde luego, los factores económicos y políticos propician y favorecen los ambientes para los cambios. Así, a la información - que siempre ha sido fundamental para la vida humana y para la sociedad- no se le había dado el lugar que hoy detenta; ahora, incluso, distingue el modelo de la sociedad del futuro: la sociedad de la información. A este respecto, Castells señala:

[...]estamos siendo testigos de un punto de discontinuidad histórica. El surgimiento de un nuevo paradigma tecnológico, organizado en torno a nuevas tecnologías de información más potentes y flexibles, hace posible que la misma información se convierta en el producto del proceso de producción [...] Las nuevas tecnologías de información, al transformar

3 Sebastián D ormido Biencomo; Julián Morales Navarro, y Luis Vicente Abad Márquez. Sociedady nuevas teendoǵas Pespetiva de desamdloindustrial. p. 113.

4 Ibídam

5 Elsa M. Ramírez Leyva. "El lector: del mundo manuscrito al cibernético". En: Lainfomaaónenlos inicios dela ea detrónica pp. 106-110. 
los procesos del procesamiento de la información, actúan en todos los dominios de la actividad humana y hacen posible establecer conexiones infinitas entre diferentes dominios, así como entre los elementos y agentes de tales actividades. ${ }^{6}$

En este sentido, cuando se habla del cambio a la sociedad de la información, se advierte la velocidad con que se transita hacia ella, por lo que sólo se lograrán adaptar las personas si se establecen las bases para que formen parte de esa sociedad, entre cuyas características figura la del aprendizaje permanente. D e tal suerte, se ha venido insistiendo en que las instituciones y organismos involucrados en procesos educativos no podrán subsistir tal y como ahorafuncionan, ya que no serán suficientes sus acciones ante los nuevos cánones tendentes a asegurar la educación para la vida como un proceso de educación continua y autodidacta con apoyo de la tecnología, a la que se supone pueden acceder todas las personas que requieren renovar los conocimientos y habilidades que demandan los ambientes laborales de esta nueva sociedad, en la que la tecnología reconfigura estructuras y relaciones en tomo de la producción y consumo de información.

Por tal razón, escuelas, universidades y bibliotecas deben participar en promover y fortalecer valores y hábitos que alejen a los lectores de caer en prácticas consumistas de información y modalidades de lectura de objetos (texto, imagen, iconos), únicamente para organizarlos sin que se logre una comprensión y asimilación cabal de esas composiciones y sólo queden a un nivel lúdico que pueden poner en riesgo procesos cognitivos que limitan el proceso de la lectura y la estructuración de formas discursivas coherentes.

Por lo anterior, debemos tomar conciencia de que la modernidad y el desarrollo de la sociedad no sólo dependen de la infraestructura tecnológica, por muy avanzada que sea y aun cuando se está convirtiendo en un parámetro para evaluar el nivel del progreso, así como de la calidad de vida de un pueblo, ya no sólo en los ámbitos científico, tecnológico y social, sino también en lo individual y en el hogar, ámbitos que ha empezado a permear.

D e tal suerte que, la tecnología de la información alcanzará de manera paulatinala mentalidad y filosofía de los pueblos, tengan éstos o no capacidad tecnológica, ya que al formar parte de las ofertas para el consumo cultural - con características como son lenguaje, equipamiento, usos y habilidades para acceder a la información-- contribuyen con elementos propios para construir una identidad personal y social en torno del paradigma tecnológico.

Esinnegable que la computadora participa en diversas actividades del individuo y se puede decir que ya ha "invadido" espacios privados y públicos en ámbitos como la educación, la investigación, el trabajo, la diversión, etc., y que en estos momentos la tecnología dela información ya es parte del grupo de las tecnologías personalesy

6 Castells Op Cit. p. 94. 
domésticas. ${ }^{7}$ D e tal forma que, asistimos a un proceso de "domesticación" de la tecnología de la información, la cual ha extendido sus efectos ala vida de instituciones y hogares, lo que sin duda redundará en un cambio de valores - aun en quienes por diversas causas no lautilizan- y de hábitos en los individuos como son, entre otros, los que tienen que ver con la lectura, las maneras de informamos y las formas de producir conocimiento.

D ada la significación social que está adquiriendo la tecnología de la información, se ha empezado a produciruna gran presión sobre ella que puede diferir de un grupo a otro hastaformar estereotipos que se extrapolan en quienes la usan y en los objetos que comunican. Ahora (como señalan Abad, D ormido y Morales), ${ }^{8}$ el problema es definir y desarrollar la tecnología acorde con el progreso de cada país, asunto complejo en un mundo de pluralidades crecientes.

Pero, como observaAntulio Sánchez, ${ }^{9}$ no por el solo hecho de entrar en contacto con la tecnología y con la información se transformará la existencia de los seres humanos; este es un proceso mucho más complejo que tiene que ver con las capacidades culturales y personales de los individuos para transformar el recurso informativo en conocimiento y éste en desarrollo, lo cual definitivamente se vincula con un factor esencial: la lectura, actividad que no se limita a un proceso intelectual sino es un proceso que pasa por la historia personal y social, por las motivaciones y el equipamiento cultural del lector. Todo ello produce diferencias en las capacidades para comprender y dar sentido a la información con la que se interactúa y para elegir la que es significativa yadecuarla alaindividualidad tanto de medios y formas textuales y gráficas, impresas y digitales, lo que en la sociedad de la información presupone que las capacidades lectoras se desarrollarán bajo otras normas determinadas por las formas físicas y las estructuras textuales digitales, aunadas a las propias de los entomos impresos.

\section{LOS EFECTOS DE LA TECNOLOGÍA DE LA INFORMACIÓNEN LOS ENTOR- NOS BIBLIOTECARIOS}

Parte importante de los efectos de la tecnología de la información es que ésta, en su carácter de medio, interviene en la construcción de tiempos y espacios diferentes de los establecidos en los medios impreso y masivos; esas dimensiones (tiempo

7 En datos que presentaEmesto López en su columna "Crece cero en el hogar, baja en empresas" en el D iario Rforma, sección semanal Intefase, del lunes 26 de abril 1999, págs. 4 y 5 A, señala que $50 \%$ de las familias mexicanas de clase media ya tiene computadora en su hogar; sin embargo, sólo 32\% están conectadas a Internet. D e 500 usuarios de computadora, sólo 35\% tiene acceso aintemeten el trabajo y $26 \%$ en el hogar. En cuanto al uso que se hace del intem\&, señala que, en primer lugar, es para utilizar el correo electrónico; en segundo lugar, para asuntos de trabajo y, en tercero, para tareas escolares e investigaciones.

8 Ibídam p.136.

9 Cfr. Antulio Sánchez. Tenitaiosuirtuales deInteme hada unnuevoconceptodela simlacón p. 65. 
y espacio), según Antulio Sánchez,10 se han convertido en cualidades aliadas de la tecnología de la información.

Las alteraciones en las dimensiones de tiempo y espacio, debidas a los grandes avances logrados en sistemas, permiten una mayor interconectividad y una mayor flexibilidad en el manejo del procesamiento de información, propiciando un conjunto de relaciones interactuantes de las que surgen nuevas y más complejas configuraciones, en cuya creación e innovación interviene el ser humano de acuerdo con su capacidad y habilidad para la selección, manejo y aprovechamiento de las nuevas estructuras informativas. Elementos determinantes en esta relación hombre-máquina son las interfaces "éstas juegan el papel de intermediarias entre la información y el usuario," 11 puesto que son las que permiten la interactividad que a su vez, permitirá mayores y más diversas posibilidades informativas en el nuevo orden de las dimensiones de tiempo y espacio.

Las características de interconectividad, flexibilidad e interactividad de las dimensiones espacio-temporales son componentes esenciales de los principios de pertinencia, oportunidad, accesibilidad y disponibilidad de la información; principios innovadores en el ámbito bibliotecario no sólo por sus procedimientos sino también por las nuevas formas de organizar y producir fuentes y servicios para responder a necesidades específicas. Actualmente podemos acceder desde diferentes lugares la información que se encuentra en un servidor, es decir, se reducen los desplazamientos físicos al lugar donde se encuentra dicha información; los usuarios se multiplican en la medida en que los servicios de información y los documentos digitales se enlazan y viajan a través de Internet yla complejidad de su manejo se reduce. Además, en la medida en que las interfaces simplifiquen los sistemas de información y los traduzcan en operaciones sencillas, el lector podrá manipular la información con mayor rapidez y alcanzar con ello una de las condiciones creadas por esta tecnología: la dimensión tiemporeal, en donde la frontera entre tiempo y espacio se empiezaa diluir, colocándonos en otradimensión. Como anotaE. Morales:

[...]en la medida en que hay conocimiento, hay información y también aplicaciones tecnológicas, y en la medida en que estas tecnologías progresan en la sociedad de la información, se vive a un ritmo acelerado: la información se genera, se transmitey se procesa a gran velocidad y además se obtiene de manera instantánea. ${ }^{12}$

10 Antulio Sánchez. Op at.

11 "Estas interfaces posibilitan la comunicación entre los sistemas de información y los usuarios en un ambiente electrónico y permiten realizar las estrategias de búsqueda para recuperar información. Para que sean consideradas como elementos de comunicación, su diseño debe basarse en los procesos cognitivos de los usuarios, sus rupturas cognitivas (problematización) ysus formas de resolver esas rupturas mediante el proceso de búsqueda y recuperación de la información".

PatriciaHernández S. "Interfaces humano/ computadora”. En:Lainformacónendiniciodelaeade trónica p.142.

12 Estela Morales Campos. "Lainformación en su iryvenir". En:Lainfomaaónenlosiniaiosdelaead@e trónica p. 23. 


\section{Investigacón Biblictedógica v. 13 No. 26 enero/ junio de 1999}

Por tal razón, las dimensiones de tiempo y espacio se consideran cualidades que cobran cada vez más significación en la biblioteca moderna y, desde luego, en la denominada biblictea digital ovirtual. A hora, incluso se piensa que lo más importante no radica en poseer los materiales informativos sino en saber dónde están y la forma de acceder a la información. D esde luego, llevar al extremo esta forma de pensar -por lo menos en estos momentos- puede implicar el riesgo de romper el equilibrio del desarrollo de colecciones documentales ya sea en su forma impresa o digital y crear vacíos informativos.

Pero es un hecho que la información ha traspasado las fronteras en las que tradicionalmente estaba contenida (página, libro, biblioteca), lo cual empieza a repercutir en el ámbito bibliotecario en cuanto a la forma de procesar y adecuar la información a las necesidades de los lectores.

El resultado de los procesos bibliográficos y bibliotecarios es una base informativa que en el medio digital (como lo menciona G eorginaA raceli Torres) ${ }^{13}$ permite re presentar el conocimiento tal y como se genera en la mente. La misma autora agrega que se han hecho propuestas encaminadas a la creación de un entorno en el que se posibilite la interacción entre el hombrey la tecnología con el propósito de ayudar al ser humano a desarrollar de manera amplia y universal sus capacidades creadoras a través del manejo de la información, aspecto que deberá tener presente el bibliotecólogo al diseñar fuentes y servicios de información.

Los lectores, cada vez más familiarizados con la tecnología de la información, intervienen en la significación social que está adquiriendo - la cual ya empieza a tener efectos en las instituciones bibliotecarias de información, de documentación y de archivo- , ya que sus expectativas y exigencias se extienden a ella "[...] porque una de las transformaciones más importantes en la base tecnológica de las sociedades contemporáneas es la que se ha denominado digitalización de la cultura," 14 y con esta digitalización, las enormes posibilidades de acceder, manipular y crear territorios informativos que son más producto de la imaginación que de la realidad.

Por lo tanto, es fundamental conocer los efectos de esta tecnología en las prácticas de lectura y de información de los usuarios, con el fin de tener bases más reales de lo que presuponemos no sólo en cuanto al surgimiento de un nuevo perfil de público sino también en cuanto a las nuevas necesidades derivadas de la tecnología de la información y los usos que de ella hagan los lectores, quienes dependerán de sus habilidades para manipularla.

Sin duda, mucho ha contribuido la biblioteca a conformar públicos y a crear nue vos ambientes informativos; sin embargo, la industria de la información, los sistemas de educación a distancia, las formas de entretenimiento virtual están favoreciendo nuevos hábitos y valores en relación con el género, la edad, la escolaridad, la

13 GeorginaAraceli Torres Vargas. “Lanavegación y exploración en lainformación digital del World Wide Web". En: Lainformacónenlosinicosdela ea detrónicap. 177.

14 AlbertoMontoya Martín del Campo. Méxicoantela reducoón teendógica p. 15. 
ocupación de las comunidades, etcétera, y el consumo de la tecnología de la información está produciendo nuevas características en los públicos.

En síntesis, con la pantallay el texto digital, del que surgen nuevas configuraciones denominadas hipetextoehipemedios se vislumbra el surgimiento de una mentalidad diferente ala que se ha estructurado con la páginaimpresa. Hipertexto e hipermedios no sólo constituyen una nueva forma de presentar lainformación y los conocimientos; se trata, como dice Landow, ${ }^{15}$ de una verdadera revolución conceptual que atañe por igual al lector, autor, profesor, estudiante y al investigador-yyo agregaría al bibliotecario-, al crearles un nuevo entorno para desarrollar sus actividades.

En estos medios ha empezado a surgir un orden -junto con el equipamiento para su manipulación- diferente de la página impresa y del libro; además, han aparecido diversidad de productos y servicios referenciales, textuales (libros, revistas, periódicos, bases de datos y diversos documentos electrónicos), gráficos, sonoros, etc., a los que se suman las Webs o páginas, las cuales se están constituyendo en una fuente de información peculiar de esta tecnología y en una entrada al denominado mundo uirtual, donde el lector se encuentra con un territorio informativo en el que sus experiencias pasadas y sus habilidades informativas le permitirán incorporar las nuevas posibilidades y requerimientos que le exige la tecnología de la información no sólo para interactuar con ella sino también para crear sus propios territorios informativos.

Al respecto, cabe mencionar que la obra de López Yépez ${ }^{16}$ Loscaminosdelainformacón: Cómbluscaryarganizar lasfuentes denuestra doumentacoón persanal, está dirigida a orientar a los ciudadanos en el diseño de una especie de centro de documentación personal sobre temas de interés, es decir, a saber organizar la información y a aprender ainformarse de lainformación, a crear nuevainformación con las ventajas que le ofrece la tecnología de interactuar con un mayor número de ofertas informativas y de realizar con ellas creaciones propias. Pero esta tarea no es fácil cuando uno se enfrenta a la creciente oferta informativa que circula en Internet. Será necesario que el bibliotecario realice un trabajo de preselección y organización como base para queel usuario personalice su propio territorio de información y, por consiguiente, será necesario desarrollar una cultura bibliotecaria en los ciudadanos dado que ésta será uno de los componentes de la sociedad de la información. El hecho de que cada individuo construya su propio territorio informativo depende de que disponga de los conocimientos, medios y habilidades para seleccionar organizar, utilizar y generar sus fuentes informativas, lo cual se está convirtiendo en condición sinequa non ya que en adelante muchas de las actividades del individuo estarán mediadas por la información digital. Este es el caso de las tecnologías aplicadas a la educación y en particular a la educación a distancia.

15 P. Landow G eorge y Patrick D ucher. El hipetexta la convergnaia dela teeńa cútica 16 José López Y épez. Loscaminosdelainfomaaón Cómosdecionaryarganizarlasfuentes denuestra doumentacoónpasonal. 
Resulta interesante que en diversos artículos sobre el tema se esté dando un lugar preponderanteal dominio de la tecnología de la información, debido a que la modalidad a distancia se fundamenta en el manejo de la información. Si bien no es nueva laidea de que lainformación es el basamento de todo proceso educativo, este asunto ha venido ocupando progresivamente espacios en los estudios sobre el tema.

Al respecto, en el informe realizado por K. Stephens ${ }^{17}$ sobre educación continua, éste señala que se olvida la educación bibliotecaria; revela, asimismo, que los estudiantes no hacen un uso suficiente de la biblioteca, y concluye que es un problema desatendido por los docentes. Además, señala que estudios realizados so bre el tema de educación a distancia revelan la necesidad de la educación bibliotecaria para los estudiantes, por lo que es imprescindible que en los estudios se integren todas las partes involucradas con el fin de articularlas con los procesos educativos, en este caso con el de la modalidad a distancia.

Por su parte, Cranor, D urndell y Primrose en su estudio 18 -realizado en la Universidad de Glasgow- sobre la enseñanza de la tecnología en el autoaprendizaje, señalan quees innegable lautilidad delas tecnologías paralos procesos deenseñanza, pero que éstas tienen implicaciones aún no previstas en su dimensión real, como son los conocimientos y habilidades que se requieren para manejar lainformación en hipertexto.

En este respecto las autoras señalan que 130 bibliotecas ya se encuentran integrando esos conocimientos como parte de la amialaque se imparte en las escuelas; se busca, en este proceso de enseñanza, un alumno más autodidacta y por consecuencia autosuficiente en el manejo de lainformación, pues, antes de la aparición de la tecnología de la información en la educación, el alumno aprendía en el libro de texto, en guías de estudio, seminarios, conferencias y a través de las dinámicas dirigidas por el profesor.

En relación con lo anterior, resulta de interés el trabajo de Barry Mahon ${ }^{19}$ quien señala que no han sido aplicados ni el conocimiento ni la experiencia bibliotecaria para crear mejores herramientas de búsqueda, tomando en cuenta las nuevas necesidades surgidas de la tecnología de la información como son los nuevos métodos para el manejo de la información en múltiples formas y en diferentes canales y modalidades de acceso.

Sin embargo, Malincolico ${ }^{20}$ agrega que los bibliotecarios han sido los primeros en adaptarse a la tecnología de la información y en utilizar los instrumentos más modernos para el manejo de la información y la comunicación. También los bibliotecarios han creado aparatos sofisticados para el uso compartido de recursos, el control

17 Kate Stephens. "The role of the library in distance learning; a review of UK, Noth American and Australian literature" En: Thenewreviewof acadamiclibraianship pp. 205-234.

18 Durndell Cranor y Primrose "Library and study skills using hypertext: the TILT experience" En: Newreview of hypemedia andmitimedia apdications andrearch pp. 127-141.

19 Barry Mahon. Eletronicinfomation 12 years of progess, What will happenin thenext 12 years?.

20 Michele Malincolico. "Librarians and innovation: An A merican view point". En: Progamdetraric andinfomationsystems pp. 447-58. 
bibliográfico y el acceso a la información así como su diseminación, apoyados en las tecnologías más avanzadas, lo cual ha sido más evidente en las dos últimas décadas de este siglo. El autor menciona que fue el sector bibliotecario norteamericano el que con gran entusiasmo entró a Internet, y de los primeros en señalar la importancia de la normatividad para el manejo de la información en la computadora y la complejidad de esas normas.

Asimismo, las bibliotecas fueron el primer mercado viable para los CD-ROMs. Por ello, se considera que sin lugar a dudas esta actitud hacia la innovación permitirá al medio bibliotecario ampliar los límites de la imaginación electrónica, la transmisión de datos, la indización automática y la vinculación del lenguaje natural con el lenguaje controlado.

Para el sector bibliotecario no es nueva la presión que ejercen sus comunidades para ver satisfechas sus necesidades informativas, las cuales, desde luego, van en proporción con el nivel de preparación y el reconocimiento del valor de la información que tenga la sociedad, de ahí el desigual nivel de desarrollo delas instituciones bibliotecarias y de información. Pero ahora, a medida que progresan los planes de los países para transitar a la sociedad de la información, estas exigencias se toman cada vez más fuertes ${ }^{21}$. Por su parte, Salvador Giner22 señala que la biblioteca debe adoptar un lenguaje universal compatible y concomitante con la pluralidad de públicos y -aquí el reto- preservando lo local. Porque las bibliotecas, nos dice el autor, estarán en el “ojo del huracán: justo en medio del conflicto entre universalismo y particularismo, cultura local y mundial, códigos morales trasnacionales e ideologías nacionales."

Además, Koenig23 señala que los efectos del avance tecnológico repercutirán en una transformación de los sistemas deinformación así como en la función que desempeñan los profesionales de la bibliotecologíay de la información. El autor propone que el acceso a la información bibliográfica y textual tenga la flexibilidad del hipertexto,

21 En el programa de trabajo para 1999, parte del programa que fue marco de la Comisión Europea, titulado Temdoǵa dela SoiedaddelaInfomadón señala en la parte denominada "Características del Programa, Contexto" lo siguiente: "En los próximos 10 años veremos el potencial de acceso universal a servicios de interés general por parte de los ciudadanos mediante dispositivos digitales de alta capacidad. El continuo progreso tecnológico permitirá que se incrementen las expectativas de los ciudadanos por lo que se refiere a servicios escalables, móviles y multimedios que respondan de forma rápida, precisa y flexible a sus necesidades y al mismo tiempo garanticen su seguridad e intimidad. El sistema nervioso de la sociedad de la información será una red de enorme capacidad y sofisticación formada por websy servidores múltiples que se solapan einterconectan bidireccionalmente en banda ancha con virtualmente cualquier nodo". Y otro de los puntos dice: "Aparecerán nuevos productos y servicios de información digitales e interactivos, y su éxito dependerá de la ca lidad y atractivo de su contenido y de la posibilidad de que los usuarios accedan a ellosy los utilicen. Eldesafío consistirá en explotar los activos creativos y tecnológicos europeos, su rico patrimonio y su diversidad así como su capital humano altamente calificado para producir, organizar, acondicionar y entregar dicho contenido. En: WWW.cordis.lu/ ist

22 Salvador Giner. "La biblioteca universal". En: LibrosdeMéxico pp. 533-56.

23 Michael Koenig. "Linking library users: A cultural change in librarianship". En:AmeicanLibraries pp. 844-849. 


\section{InvestigadónBiblictedógica V. 13 No. 26 enero/ junio de 1999}

que los datos proporcionados por el usuario predominen sobre el bibliotecario o los editores; en suma, que el diseño y operación de herramientas de búsqueda incluyan formas de interacción con la información que suministran los sistemas.

En cuanto a dichas herramientas, quizá sea conveniente analizar con más detenimiento la propuesta de Bierbaum ${ }^{24}$ respecto de "la ley del menor esfuerzo", implícita en las leyes de Ranganathan, Cutter y en la ley de Zipf: Humanbehaviorand theprinapleofleasteffat. En la ley de Mooers se observa que "un sistema de recuperación de información no será utilizado cuando el obtener la información signifique para el usuario más problema que no tenerla”. En el estudio de Pool se encontró una ligera diferencia entre la tendencia al menor esfuerzo y la reticencia a la dificultad en la recuperación de información entre los científicos.

E s importante señalar que indudablemente la tecnología de la información soluciona algunos de los problemas que han aparecido con la explosión de la información impresa; sin embargo, ahora surgen nuevas necesidades, lo cual es normal ya que lo mismo sucede con toda nueva tecnología; esta situación plantea para la biblioteca el desarrollo de un nuevo ambiente de enseñanza, aprendizaje, información y hasta de recreación, ya que el flujo de conocimientos, ideas y sucesos estará cada vez más involucrado con lavida cotidiana. Por ello, estanueva tecnología tendrá que ofrecer alternativas que simplifiquen los procesos que requieran de una inversión excesiva de esfuerzo y tiempo por parte de los usuarios; es decir, estamos ante un cambio de perspectiva, ahora la atención debe ponerse en las formas en que se busca la información más que en la transferencia.

Estos puntos de vista deberán estar correctamente contrastados con el lector actual, pues en estos momentos es fundamental conocer las repercusiones que ha tenido la tecnología de la información en sus actitudes, hábitos, valores y prácticas nuevas surgidas de cara a los nuevos medios y formas de información. Al respecto se presentan a continuación los resultados de algunos estudios que se han realizado en cuanto a los efectos de la tecnología de la información en los lectores.

En los países donde el uso de computadores se ha incrementado es indudable que sus usuarios están cada vez más acostumbrados a utilizar poderosos procesadores de texto que de alguna manera lo s hafamiliarizado con las capacidades de las máquinas y programas; algunos sectores también se han familiarizado con los CDROMs, catálogos electrónicos, diccionarios, enciclopedias, revistas, Internet, páginas Web y, aun en diversos niveles de dominio, el usuario percibe el potencial de la tecnología de la información en cuanto ala interacción con lainformación; más aún, en algunos grupos se han dado cambios significativos en la obtención de información, en las formas de comunicación, de hacer negocios o de divertirse mediante la tecnología de la información.

24 Cfr. Esther G. Bierbaum. "Save the time of the reader: A paradigm for the 90s". En: AmmicanLibravies pp. 18-19. 
* E.d. D. Smith, ${ }^{25}$ en su estudio realizado en instituciones académicas: A com parison of theeffets of neutechndogesonserchinghabitsinindustrial andacademicins titutions, encontró que el uso de bases de datos en chromprácticamente ha sustituido las búsquedas en línea. La razón que dieron los usuarios es que se sentían más relajados y para algunos hasta resultaba divertido. Por su parte, los bibliotecarios entrevistados señalaron que el cotromles facilitaba la tarea de orientar a los usuarios para que realizaran sus búsquedas. En conclusión, el autor señala que el colromha adquirido arraigo en los medios académicosy especializados y se utiliza como una fuente más de información.

- Respecto del uso de Internet se publicó el estudio A companisanofrangeandvar lued use of theintemet withtraditional referencesarcesin Scetchpubliclibraries, basado en una encuesta realizada en 1995 por el D epartamento de Bibliotecas y la Información en red de G ran Bretaña, con el fin de asesorar al gobierno de ese país sobre el uso de Internet en las bibliotecas públicas. En el informe se señalo que estas bibliotecas estaban destinadas a jugar un papel importante en la revolución de la tecnología de la información, al proporcionaral mayor número de personas el máximo acceso al conocimiento y a la información almacenados electrónicamente. Entre los aspectos que se analizaron, está el control de calidad y el gran volumen de registros manejados. Las ventajas de Internet para dar solución a estos problemas descansan, sin embargo, en las habilidades de los especialistas para recuperar la información. Se considera que la capacitación ya no debe limitarse a las técnicas de búsqueda y recuperación; ahora es indispensable que los especialistas en consulta también cuenten con los conocimientos básicos para atender problemas técnicos del manejo de esta herramienta.

- En un estudio A statevideevaluationoftheimpactoftechndogyintegation, ${ }^{26}$ levado a cabo para analizar el impacto que ha tenido la tecnología entre profesores y estudiantes de 80 instituciones educativas del estado de Idaho, EUA, los autores señalan que en ese estado se ha legislado con objeto de crear las condiciones óptimas en las instituciones educativas, lográndose la integración masiva de la tecnología al currículum. Los profesores han adquirido suficientes capacidades no sólo para el manejo de las nuevas tecnologías sino también para crear nuevos desarrollos con el fin de integrarlos de manera eficaz a sus programas. Hasta ahora, los docentes han observado resultados favorables de sus alumnos en cuanto a las actitudes positivas en el proceso de aprendizaje y, algo que compete a nuestro ámbito es que la tecnología está dando gran realce a las bibliotecas escolares. G eneralmente en estos estudios está presente la idea de los efectos de la tecnología en los cambios en

25 Jaumal of Information Saiene pp. 57-66.

26 D ebra D irkinsen,Bauer Christine, Coffland D avid, Sarah Naylor..A statevideealuation of theimpact fteehndogyintegation En:http/ www.coe.uh.edu/ insite/ elec pub pub/ HTML 1998/ redirkhtm. 
actitudes y prácticas, y por lo regular se tiene una opinión positiva ya que se logran mejores resultados en los procesos educativos y en el manejo de información y comunicación.

En un estudio sobre actitudes de profesores y estudiantes de la carrera de periodismo respecto de la tecnología de la información se encontró que según la etapa de asimilación de la tecnología en las categorías que van del convencido y entusiasta de la tecnología al escéptico, todos, de alguna manera, consideran que con la nueva tecnología se dan cambios en las formas de obtener conocimiento y comunicarse.

\section{LA TRANSFORMACIÓN DEL ÁMBITO BIBLIOTECOLÓGICO EN LA SOCIEDAD DE LA INFORMACIÓN}

Sin duda, la lectura ocupa un espacio de vital importancia en el ámbito bibliotecológico, dado que es el proceso mediante el cual losindividuos se apropian de los contenidos del mundo documental, sistematizado conforme al orden establecido por los métodos y técnicas editoriales, bibliográficas, y bibliotecarias las cuales permiten acceder a ese mundo documental a través de formas institucionalizadas, que en bue na medida han contribuido a crear, fortalecer y socializar modalidades de lectura y de información como parte de la cultura impresa que aún prevalece.

Sin embargo, en los entornos laboral, educativo y de esparcimiento es una realidad el incremento de ambientes informativos abundantes y variados tanto en el aspecto de sus componentes - textos, imágenes fijas y en movimiento, sonido- , como en calidad y presentación, con características interactivas, acceso remoto y flujos informativos que hacen posible la tecnología a través de las redes.

Ante esta situación, las formas institucionales, incluso aquellas que han dado contexto a estas tecnologías, están siendo ahora cuestionadas y se les está exigiendo una profunda revisión de sus estructuras para transformarlas en entornos más fluidos por donde transite la información y sea utilizada por los diferentes sectores sociales no sólo locales sino de todo el mundo.

En este contexto, es importante que analicemos con todo cuidado las perspectivas sociales y humanísticas de nuestro ámbito bibliotecológico que, hasta ahora, se ha vislumbrado en relación con la tecnología de la información. Una de dichas perspectivas asegura que los niveles de desigualdad de desarrollo se reducirán debido a que en el futuro será posible que una proporción cada vez mayor de las comunidades acceda a la información, lográndose así avances en el terreno educativo, científico, cultural, con la posibilidad de difundir conocimientos a sectores y sociedades marginados o con recursos limitados, y que éste será el camino para mejorar la calidad de vida de todos.

También se afirma que las formas institucionales actuales tendrán que transformarse o ser sustituidas por estructuras en red, cuya vitalidad estará basada en 
la comunicación y en lainformación sostenida por todos, mediante la base tecnológica desde luego, en donde cualquier individuo podrá compartir información para realizar diversas actividades, al grado que se espera lograr una suerte de democracia perfecta: todos contribuyen y todos se benefician de la información. ${ }^{27}$

En otros casos se considera que la información se está convirtiendo, precisamente, en una mercancía con el suficiente poder para desarrollar prácticas, hábitos, valores sociales y mentalidades destinados a propiciar conductas más bien consumistas con el fin de sostener laindustria tecnológica de lainformación y de la telecomunicación y que, por otra parte, se adecuan a las exigencias de formación y capacitación que requieren los entornos laborales, con el riesgo producir masas desinformadas, cuando no estén debidamente preparadas para seleccionar y utilizar la información en su desarrollo.

De manera inversa, otra perspectiva propone que las instituciones educativas - cabe sumar las bibliotecarias- , entre otras, transformen sus estructuras en relaciones flexibles, donde la tecnología de la información se contextualice dentro del proceso de enseñanza aprendizaje mediada por el conocimiento. Es decir, que estas instituciones fortalezcan su presencia en la sociedad y que contribuyan realmente a la formación deindividuos, reduciendo con ello las diferencias relacionadas con el derecho a la información y a la lectura en el medio impreso y ahora en el digital.

En cualquier caso, se debe buscar que la lectura sea el proceso mediante el cual se reproduzca un discurso cuyo contenido manifieste la selectividad, comprensión, crítica e integración de los conocimientos con los actuales de creación, innovación y originalidad, Es decir, que las escuelas, universidades y bibliotecas contribuyan a la formación de individuos capaces de utilizar lainformación y crear conocimiento, yno busquen el dominio del manejo de la tecnología como un fin sino como un medio que permita al individuo expandir sus habilidades y creatividad, a tal grado que logre, con una mentalidad para la innovación, participar en el crecimiento de su entorno.

De lo contrario, se puede producir una acumulación de información que no sea debidamente asimiladay se reduzca a la mera repetición de fragmentos informativos y, más que conocimiento, se produzca desinformación, pues están surgiendo otros escenarios - paralelos a escuelas, universidades y bibliotecas- que ofrecen programas de formación y actualización a distancia con tutores electrónicos basados en inteligencia artificial, multimedios así como de servicios deinformación, es decir, puede darse el caso de que la tecnología ahora contextualice esas actividades, las cuales se realizan a manera de autoservicio y masivamente, con las que se pretende substituir

27 En 1948, Norbert Wiener introduce la idea de la sociedad de la información y señala la transferencia de la información como el elemento que permitirá ejercer la democracia, incluso se habla de que la telecomunicación interactiva producirá una teledemocracia y la sociedad tendrá acceso a casi las mismas fuentes de información que los gobernantes y las decisiones, ya sea directa o indirectamente, se tomarían en conjunto.

Cfr. Víctor Flores O lea y Rosa Elena G aspar de Alba. Intemeyla reuduiónaibeméica. p. 42. 


\section{InnestigacónBiblictedógica V. 13 No. 26 enero/ junio de 1999}

al profesor por los medios tecnológicos para formar y capacitar recursos humanos adecuados alas exigencias del mercado laboral y obtener con ello altos rendimientos económicos.

La respuesta de la bibliotecología a esta nueva circunstancia que coloca a la información como mercancía y que puede marcar pautas de consumo más que un proceso de aprendizaje, como se pretende en la sociedad de la información, tendrá que sostener la posición que hasta ahora ha defendido: la información como parte de un bien para el servicio público. D esde luego, los medios físicos en donde se materializa el conocimiento siempre han sido una mercancía, pero la institución bibliotecaria los ha transformado en instrumentos y servicios públicos para la lectura y la información. A demás, es importantedestacar que una de las funciones fundamentales de las bibliotecas del futuro es seguir asegurando el espacio físico en el que se mantiene el conocimiento de la cultura escrita, lo cual incluye las formas originales en las que se haya materializado el conocimiento; también comprende las que se hayan transferido, los actuales medios electrónicos y los que se produzcan de aquí en ade lante, es decir, el patrimonio documental en cualquiera de sus formas, como parte del patrimonio cultural, base para ejercer el derecho de cualquier pueblo a la información y a la lectura.

La institución bibliotecaria es la que ha contribuido de manera plena a ejercer ese derecho, sometido no pocas veces a los mandatos ideológicos a través de límites presupuestales o de censura. Al parecer, una de las particularidades dela sociedad dela información es extender la disponibilidad de la información a todos los ciudadanos, pero deberán fortalecerse las políticas de financiamiento y equipamiento para asegurar uno de los fines de la sociedad del conocimiento, la información para todos.

Al respecto, se tiene la expectativa de que la tecnología de la información abrirá candados impuestos al flujo de lainformación al suponer quela digitalización es una suerte de llave que hace posible la disponibilidad universal todos los textos pasados, presentes y futuros, que cualquier lector, en donde se encuentre, que tenga acceso a un equipo conectado a una red, tendrá la facilidad de acceder a una suerte de biblioteca universal. Pero esta es unailusión creada un tanto por la imaginación y otro tanto por la industria de la información. Actualmente, existen limitaciones de carácter legal (leyes de derechos de autor) y comercial para trasladar la producción impresa al medio digital.

El surgimiento del lector remoto plantea una revisión de los compromisos que la institución bibliotecaria tiene con esta nueva modalidad de público, ya que forma parte de un mundo globalizador en el que, por un lado, domina la idea de compartir los recursos - en este caso informativos- pero, por otro, la de inversión y recuperación de costos.

En los ambientes bibliotecológicos se ha empezado a revisar y proponer métodos, técnicas y posiblemente principios, dando un lugar preponderante al aspecto social y humanístico, dado que ha inquietado el excesivo entusiasmo por la tecnología de la información como la gran panacea que resolverá los viejos problemas 
bibliotecológicos, en cuanto a orden, control y disponibilidad de los recursos documentales como parte de los entornos que favorezcan el logro de una sociedad lectora e informada.

Pero esta tecnología sin duda agrega una nueva dificultad a las que ya de por sí tie ne la cultura impresa y que es preocupante porque sus efectos alcanzan a los que por distintas razones no pueden integrar la tecnología de la información a sus practicas, habilidades y, posiblemente, ni siquiera a su mentalidad. O tros asimilan en diferentes grados los cánones que empiezan a establecerse en lainteracción con la pantallay el medio digital - lectores cuya formación, actividad laboral y esparcimiento están mediados por la tecnología de la información y que han empezado a modificar o a crear nuevas prácticas y necesidades de lectura y de información.

Como ya lo ha señalado Chartier, 28 la lectura del texto electrónico jamás será lo mismo: la lectura puede someter al texto a múltiples operaciones (indizar, anotar, copiar, deshacer, recomponer, transferir); más aún, el lector puede asumirse como coautor, puesto que en todo momento puede intervenir en el texto, modificarlo, reescribirlo, darle sentido. Así, los textos en el medio digital se pueden multiplicar tantas veces como lectores y versiones se lancen al ciberespacio.

A la variedad de medios impresos se suma la documentación electrónica, objeto de un tratamiento editorial y bibliotecológico diferente a los impresos. Ante ello, la bibliotecología - disciplina cuyo cuerpo de conocimientos se ha desarrollado históricamente en función de los principios y operaciones que han hecho posible ordenar el mundo de los textos conforme las sociedades se democratizaban-, ahora empieza a proponer estructuras de organización para ordenar la información que transita en las redes infoespaciales.

Se requerirá un nuevo orden ahora que las colecciones ya no obedecen a estructuras cerradas; a diferencia de antes, los sistemas clasificatorios tendrán que ofrecer alternativas para enlazarse no sólo a las colecciones sino también a instituciones bibliotecarias para su reconfiguración y servicio en red, con base en la tecnología de la información, y el diseño de herramientas y servicios bibliotecarios para el acceso a la información atípica e inconmensurable que transita por las redes ciberespaciales, en donde la biblioteca sin paredes sigue vigente en su función socializadora del mundo de la información sistematizada para los lectores de libros y para los lectores de los medios digitales, para quienes las nuevas modalidades de lectura, surgidas de la cultura cibernética, ofrecen una proliferación de información que parece hacerse infinita, y las facilidades para acceder a ella provocan más ago bio en la medida en que se multiplican las ofertas informativas. Paradójicamente, en tanto se reduzcan las dimensiones espacio temporales, que se privilegian en el ambiente digital, provocarán un consumo mayor de tiempo para la revisión y selección de ofertas.

28 Roger Chartier. "Le message écrit et ses receptios. D u codex à l'écran". En: Quadami di sudi samidia. p. 36. 


\section{Investigacón Biblictedógica V. 13 No. 26 enero/ junio de 1999}

Y todavía más, las estructuras clasificatorias deberán contemplar las necesidades que tienen los lectores para ordenar sus ambientes informativos particulares, -que solamente se limitan al control documental- sino también aquellas que les facilite el establecimiento y actualización constante de sus redes personales.

Actualmente la bibliotecología, como disciplina, debe buscar alternativas para formular una culturabibliotecaria entre los ciudadanos, a pesar de lo que señala Blaise Cronin ${ }^{29}$ en el sentido de que más que invertir tiempo y recursos en la formación de usuarios se deben desarrollar alternativas para facilitar el acceso y la interacción entre la sociedad y la información; desde luego, no se puede soslayar la obligación de los bibliotecólogos en esa tarea.

Aunque la industria se ha venido procurando, como un elemento de competitividad entre las compañías, que sus productos sean "amigables", esto no es suficiente. Por ello, el bibliotecólogo debe, al menos en la actualidad, llevar a cabo acciones enérgicas e insistentes para la formación de lectores aptos en el uso de la información.

Es más, la forma en que la bibliotecología puede contribuir es constituyéndose como guía del lector - función que anteriormente se compartía con el docente- ante el riesgo, como ya se mencionó anteriormente, defortalecer una modalidad de lectura más que de contenidos de objetos de las formas hipertextuales (en tanto es una obrafragmentada, lejos del control del autor), en donde la profundidad la dan las relaciones que se establezcan a través de la red y el medio tecnológico. La fragmentación de la obra remarca la ausencia del autor, quien hacía las veces de autoridad, del maestro que nos enseña el camino para adentrarnos a un conocimiento conforme a la secuencia que le ha dado a su obra.

En la sociedad actual y en la de la información la bibliotecología podrá desempeñar funciones muy destacadas no sólo en lo que compete al orden documental para asegurar su disponibilidad y accesibilidad de manera pertinente y oportuna. En los procesos educativos en donde la tecnología de la información, como hemos visto y se ha mencionado, empieza a tener una presencia importante, la cultura bibliotecaria es ya indispensable en el currículum de escuelas y universidades, puesto que se avizoran cambios en los actores de la actividad docente en donde profesores y estudiantes son, como señala Jordi Adell,30 agentes activos en la búsqueda, selección, procesamiento y asimilación de la información.

A hora, en la denominada sociedad dela información, que se ha empezado a proponer como prototipo demodernidad, de modelo a seguir en el próximo milenio, se producirán procesos sociales destinados a generar conocimiento y aprendizaje permanente a lo largo de toda la vida, de tal suerte que la lectura será el medio indispensable

29 Cfr. Ramírez Leyva. "Los recursos humanosylos usuarios". En:Labiblidteadd futuro pp. 165-170.

30 Jordi Adell. "Tendencias de la educación en la sociedad de las tecnologías de la información”. En: EDITEC, Reista detrónica edurativa.

(http/ / nti.iji.ed doss/ nti/Jard_Adell_EDUTEC.htlm) 
para transformar la información en desarrollo y bienestar social y con ello identidad, pertenencia y promoción en este nuevo tipo de sociedad.

La gran interrogante es si esta nueva tecnología de la información ejercerá alguna fascinación que logre transformar la lectura por obligación en lectura por convicción en la población, en general, y en los sectores instruidos, en particular, dado que estos últimos no lo han logrado aun cuando la cultura impresa los ha dotado con equipamiento cultural (educación, bibliotecas, libros, publicaciones periódicas, entre otros).

\section{BiBLIOGRAFÍA}

Adell, Jordi. “Tendencias de la educación en la sociedad de las tecnologías de la información". En: EDITEC, Revista dectrónica eeturativa. Nov 1997: (7) (http:/ / nti.ji.es/docs/ nti/Jordi_Adell_EDUTEC.htlm)

Bierbaum, Esther G. "Save the time of the reader: A paradigm for the $90 \mathrm{~s}$ ". En: AmericanLibranes1990, vol. 21, núm. 1, págs. 18-19.

Castells Manuel. Laeradelainfomadón Econmá, soiedadyailtura, vol. 1: Laso decladred México : Siglo XXI, 1999.

Cranor, D urndell y Primrose "Library and study skills using hypertext: the TILT experience". En: Newreviewof hypemedia andmutimedia apdicationsand rearch vol. 2, 1996, pags. 127-141.

Chartier, Roger. "Le message écrit et ses receptios. Du codex à l'écran". En: Quadami di sudi semidid, 1995 núm. 72.

D irkinsen, D ebra,,BauerChristine, Coffland D avid, Sarah Naylor..A statewide evaluation of theimpad of techndogyintegration En:

http/ www.coe.uh.edu/ insite/ elec pub pub/ HTML 1998/ re dirk htm.

D ormido Biencomo, Sebastián; Julián Morales Navarro, y Luis Vicente Abad Márquez.Sociedad y nueras teendoǵas Pespeetiva dd desamolloindustrial. (Col. Estructuras y Procesos. Serie Ciencias Sociales) Madrid: Editorial Trotta, 1990.

Flores O lea,Víctory Rosa Elena Gaspar de Alba. Intemeylareuduaónábemátca. México, 1997.

Giner, Salvador. "La biblioteca universal". En: LibrosdeMéxica, 1993 : (32), págs. 533-56.

Hernández S., Patricia "Interfaces humano/ computadora”. En: Lainfomadớnendiniciodelaeradectrónica, vol. 2, México: UNAM, 1998. págs. 139-155.

Koenig, Michael. "Linking library users: A cultural change in librarianship". En: AmericanLibraies 1990, vol. 221, núm. 8, págs. 844-849.

Landow G eorge P. y Patrick D ucher. El hipetexta laconvergenaadelateenáaútica. España : Paidos, 1995.

López Emesto. "Crece cero en el hogar, baja en las empresas" en el Diario Re forma, sección semanal Intefase, del lunes 26 de abril 1999. 
López Y épez, José. Loscaminosdelainfomadón Cómosdecianaryar ganizar las fuentes denuestra dbumettacón personal.

Mahon, Barry. Eletronicinformation: 12 years of progess; What will happeninthenext 12 years? Congreso de D ocumentación Automatizada. Cáceres, España, 1996.

Malincolico, Michele. "Librarians and innovation: An American view point". En: Progameetronicandinformationsystems vol.31, (1), pags. 447-58.

Montoya Martín del Campo, A lberto. Méxicoantelarevduouónteemo lógica ed. Diana, 1993.

Morales Campos, Estela. "La información en su ir y venir". En: La infomaaón en los inicios dela era detrónica, vol. 2. México: UNAM 1998.

Programa de Trabajo para 1999 de la Comisión Europea. Tendo ga dela Scoiedaddelainformaión WWW.cordis.lu/ ist

Ramírez Leyva, Elsa M. "El lector: del mundo manuscrito al cibernético”. En: Lainfomaaón enlosiniaiosdelaera dectrónica, vol. 2. México: UNAM, 1998, pags. 106-110.

Ramírez Leyva. "Los recursos humanosy los usuarios". En:Labibicteca de futuro UNAM, México, 1996, pags. 165-170.

Sánchez, Antulio. Teritcriosuirtuales deIntemethaiaunnuevoconeqto dela simlacoón México: Taurus, 1997.

Silverstone, Roger y Hirsch, Eds. Losfeetosdelanuevacammicacoón d consumdela nueva teendoǵa en d hogaryla familia1996.

Smith, Eduard D. "A comparison of the effects of new technologies on searching habits in industrial and academic institutions”. En:Jaumal ofinformationsienęvol. 19, 1992, págs. 57-66.

Stephens, Kate. "The role of the library in distance learning; a review of UK, Noth American and Australian literature". En: The newreview of academiclibraniandip, v. 2 , 1996 pags. 205-234.

Torres Vargas, G eorgina Araceli. "La navegación y exploración en la información digital del WoddWideWeb”. En: Lainfoma aónenlosiniaiosdelaeradetrónica, vol. 2. México: UNAM, 1998. 